\title{
Demographic consequences of reproductive interference in multi-species communities
}

\author{
Janice J. Ting and Asher D. Cutter ${ }^{*}$ (D)
}

\begin{abstract}
Background: Reproductive interference can mediate interference competition between species through sexual interactions that reduce the fitness of one species by another. Theory shows that the positive frequency-dependent effects of such costly errors in mate recognition can dictate species coexistence or exclusion even with countervailing resource competition differences between species. While usually framed in terms of pre-mating or post-zygotic costs, reproductive interference manifests between individual Caenorhabditis nematodes from negative interspecies gametic interactions: sperm cells from interspecies matings can migrate ectopically to induce female sterility and premature death. The potential for reproductive interference to exert population level effects on Caenorhabditis trait evolution and community structure, however, remains unknown.

Results: Here we test whether a species that is superior in individual-level reproductive interference (C. nigoni) can exact negative demographic effects on competitor species that are superior in resource competition (C. briggsae and C. elegans). We observe coexistence over six generations and find evidence of demographic reproductive interference even under conditions unfavorable to its influence. C. briggsae and C. elegans show distinct patterns of reproductive interference in competitive interactions with C. nigoni.

Conclusions: These results affirm that individual level negative effects of reproductive interference mediated by gamete interactions can ramify to population demography, with the potential to influence patterns of species coexistence separately from the effects of direct resource competition.
\end{abstract}

Keywords: Caenorhabditis, Reproductive interference, Interference competition, Sperm

\section{Background}

Resource competition, predation, and mutualism/ parasitism span the gamut of traditional views about ecologically-important interspecies interactions [e.g. 1-5]. Interspecies sexual interactions like misdirected courtship, heterospecific mating, and hybridization, however, may not just be 'noise' [6]. Instead, reproductive interference (RI) can result from reduced fitness in one or both species through direct harm, wasted time or energy, or forfeited gametes from interspecies sexual interactions as a form of interference competition

\footnotetext{
*Correspondence: asher.cutter@utoronto.ca
}

Department of Ecology \& Evolutionary Biology, University of Toronto,

Toronto, ON M5S3B2, Canada
[7-9]. Similar to other interspecies interactions, RI can influence community structure and evolutionary trajectories from species displacement versus coexistence, specialization (e.g. spatial, temporal, or habitat), and reproductive character displacement [7, 10, 11]. Evolutionary responses of species to harmful sexual interactions between them thus can represent an interspecies byproduct of intraspecies sexually antagonistic evolution [12]. Even if interspecies sexual interactions affect individual fitness ('component RI'), however, theory shows that community characteristics are not guaranteed to be perturbed ('demographic RI') [10]; if demographic RI occurs, however, then some mechanism of component RI must also be present [10]. Thus, it is crucial to determine the incidence of both component and 
demographic RI to understand the potential for harmful interspecies sexual interactions to influence population dynamics and community composition.

To predict whether or not species can coexist with one another, it is important to consider both reproductive interference and resource competition. In competitive dynamics, the defining characteristic of RI is its positive frequency dependence: the rarer species will suffer disproportionately from costly interspecies sexual interactions [13]. As a result, species coexistence becomes less likely even with weak effects of RI [13-15]. The outcome of this frequency dependent effect of RI is simplest to conceive when the costs of RI are symmetric between species, rather than one species suffering disproportionately. Whether species can coexist with one another, however, depends especially strongly on the degree of the asymmetry of RI compared to the asymmetry of resource competition [16], and RI is typically observed in empirical studies to be asymmetric, where one species is more adversely affected than the other [7]. As a result, a species that is superior in resource competition could coexist with or even be excluded by another species that is superior in reproductive interference [16]. This dynamic is especially pertinent to modern-day communities because, for example, conditions favourable for biological species invasions due to global climate change may set the stage for both resource competition and reproductive interference to contribute to the likelihood of species invasion success $[7,17,18]$. Human impacted environments also may aggravate the incidence of interspecies sexual encounters by disrupting pre-mating cues (e.g. noise and light pollution on courtship $[19,20])$.

Reproductive interference often is studied from the perspective of hybrid progeny production between species, though mating and pre-mating modes of RI also have been characterized (signal jamming, heterospecific rivalry, misdirected courtship, heterospecific mating attempts, erroneous female choice) [7, 21, 22]. Incompatibilities between species at the gametic level also are known to make important contributions to the speciation process [23]. Consequently, reproductive interference also could manifest at the gametic level, during the postmating pre-zygotic phase, contributing to component RI, or demographic RI, or both. If females of a species commonly suffer harm from having mated with males of other species, then we would expect selection to favor the evolution of tolerance or resistance to the harm, or, of pre-mating barriers to preclude exposure to the negative effects, as for reproductive character displacement [9, 24]. Thus reproductive interference has the potential to drive within-species mate choice dynamics as an evolutionary outcome of between-species interactions.
Failure of species recognition from weak mate preference/avoidance in Caenorhabditis nematodes leads different species to mate with one another and transfer sperm [25-28]. These interactions can exact harm on female individuals, inducing sterility and reduced longevity [28], thus demonstrating component RI. The primary source of this individual-level reproductive interference is heterospecific male sperm that displace existing conspecific sperm and that migrate to ectopic regions of the gonad or even into the body cavity [28]. This form of gametic reproductive interference does not affect all species equally (asymmetrical reproductive interference) and has no known effect on males (sex-biased) [28]. Moreover, Caenorhabditis mating, even with conspecific males, can be costly in the form of reduced longevity and lifetime reproductive output $[29,30]$, for example, with mating-induced physical damage to the cuticle from repetitive spicule insertions by males that potentially increase vulnerability to bacterial or fungal infections [31, 32]. Most species of Caenorhabditis are dioecious (male/female), with three known exceptions, including C. elegans and C. briggsae used in this study, in which populations are androdioecious (males and self-fertile hermaphrodites; hermaphrodites incapable of inseminating other hermaphrodites) [33]. In addition, experimental populations of $C$. elegans and C. briggsae can be genetically manipulated to be either purely hermaphroditic or fully dioecious [34]. Purely hermaphroditic populations typically have much higher growth rates than mixed-sex populations [35], potentially leading to displacement or extinction of outcrossing species through simple competition for resources. However, such species would represent disproportionate targets of sperm-induced harm by virtue of the female morphology and sensitivity to heterospecific harm of hermaphrodite individuals [28], such that demographic RI could disadvantage them despite their superior resource competition $[18,36]$. In principle, such interactions could contribute to patterns of local and regional species richness in these organisms that have highly patchy resource distributions [33, 37] and potentially drive the evolution of mate choice traits. Here we test for multi-generation demographic effects of asymmetric gametic RI in Caenorhabditis nematodes by quantifying community composition and population growth rates in experimental species assemblages.

\section{Methods \\ Overview}

We created experimental communities comprised of one or two species of Caenorhabditis nematodes and quantified their abundances after $\sim 6$ generations (15 days); we use the term 'community' broadly to define groups of organisms of different species or of the same species 
that differ in a fundamental reproductive phenotype that share common resources and habitat. We distinguished species with the aid of integrated transgenic fluorescent markers expressed in pharyngeal muscle (green fluorescent protein, GFP, in C. elegans and C. briggsae; Discosoma sp. red fluorescent protein, DsRed, in C. nigoni; Table 1). By rearing the animals with ad libitum food availability, we minimized resource competition with the aim of testing for interference competition from the sensitivity of population growth rate estimates to the presence of other species [16]. Specifically, interspecies matings of $C$. nigoni males to C. elegans and C. briggsae lead to ectopic migration of sperm that induces sterility and premature death of inseminated individuals [28], termed 'component reproductive interference (RI),' which we hypothesized could depress population growth rates of C. elegans and C. briggsae in mixed-species communities ('demographic RI'). Females of $C$. nigoni do not suffer detectable component RI effects [28]. All three species also exhibit strong post-zygotic reproductive isolation with one another [38-41]. By using isogenic and isofemale strains for each species, we limit the communities to ecological dynamics and exclude potential coevolutionary responses.

\section{Experimental community composition}

We constructed seven community types for each of two experimental blocks that involved either C. elegans or C. briggsae. Two of the treatments types contained just a single isogenic strain of a single species (Table 1): wildtype C. elegans (PD4790, GFP transgene marker in $\mathrm{N} 2$ genetic background) or wildtype C. briggsae (JU610, GFP transgene marker in AF16 genetic background) and C. nigoni (VX0090, DsRed transgene marker in JU1325 genetic background; repeated in both experimental blocks). C. elegans and C. briggsae populations are normally composed entirely of self-fertilizing hermaphrodites whereas C. nigoni populations are dioecious with an equal sex ratio of males to females.

The remaining five treatment types contained twostrain communities, such as wildtype $C$. elegans hermaphrodites mixed with C. nigoni (Table 1). Gonads of hermaphrodites first produce sperm in both C. elegans and $C$. briggsae, then switch to irreversible production of oocytes which enables self-fertilization [42]. Hermaphrodites are incapable of inseminating other hermaphrodites, and their behavior leads to poor mating efficacy even with conspecific males ( $<1 \%$ male individuals) [27]. In communities containing these hermaphrodites mixed with heterospecific $C$. nigoni, therefore, we expect only a fraction of hermaphrodites to mate and receive harmful C. nigoni male sperm.

To experimentally increase the potential exposure of C. elegans and C. briggsae to harmful heterospecific male sperm, we constructed mixed communities containing 'feminized' hermaphrodites with an allele that transforms hermaphrodites into 'females' (Table 1). The C. elegans isogenic strain JK574 thus is dioecious with a 1:1 male:female sex ratio (fog-2 knockout mutation in N2 genetic background) [35, 43]. Consequently, the females must mate to reproduce which may increase their risk of erroneous heterospecific mating and reproductive interference (similar feminizing effects for C. briggsae strain VX0262 with disruption of Cbr-she-1 in an AF16 genetic background) [44]. To complement the interspecies community type, we also constructed communities comprised of wildtype and feminized strains of the same species for both C. elegans and C. briggsae (e.g. PD4790 with JK574; Table 1).

With the aim of creating an experimental treatment that minimizes the potential for sperm-mediated reproductive interference, we constructed communities of C. nigoni mixed with hermaphrodites of C. elegans or C. briggsae that are incapable of mating (Table 1).

Table 1 Species and strain genotypes used to create experimental communities

\begin{tabular}{|c|c|c|c|}
\hline Species & Strain: genotype ${ }^{a}$ & Phenotype & $\begin{array}{l}\text { Tendency } \\
\text { to receive male } \\
\text { sperm }\end{array}$ \\
\hline C. briggsae & JU610: mfls5[Cbr-egl-17::GFP; Cel-myo-2::GFP] & GFP-marked 'wild type' facultative outcrossing hermaphrodites & Moderate \\
\hline C. briggsae & VX0262: Cbr-she-1(v83) & 'Feminized'females and males & High \\
\hline C. briggsae & DY199: Cbr-lin-39(bh23) & 'Vulvaless' selfing hermaphrodites & Low \\
\hline C. nigonib & VX0090: mfls42[Cel-sid-2; Cel-myo-2::DsRed2] & DsRed-marked females and 'harmful' males & High \\
\hline C. elegans & $\begin{array}{l}\text { PD4790: mls12 II [myo-2p::GFP + pes- } \\
\text { 10p::GFP + F22B7.9p::GFP] }\end{array}$ & GFP-marked 'wild type' facultative outcrossing hermaphrodites & Moderate \\
\hline C. elegans & JK574: fog-2(q71) & 'Feminized'females and males & High \\
\hline C. elegans & PS436: let-60(sy93) & 'Vulvaless' selfing hermaphrodites & Low \\
\hline
\end{tabular}

a Cbr-she-1 [44], Cbr-lin-39 [80], PD4790 [81], fog-2 [82], let-60 [55]

b Same isofemale strain genotype of $C$. nigoni was used in both experiments as the source of heterospecific male sperm 
Specifically, we used isogenic strains of hermaphrodites that lacked a vulva and so can only produce self-progeny because they cannot be inseminated (C. elegans PS436 with genetic disruption of let-60 in the N2 genetic background; C. briggsae DY199 with genetic disruption of Cbr-lin-39 in the AF16 genetic background; Table 1). A consequence of the vulvaless phenotype is that eggs will hatch inside the hermaphrodite, leading to lower fitness and longevity than wildtype hermaphrodites [45]. To complement the interspecies community, we also constructed communities comprised of wildtype and vulvaless strains of the same species for both $C$. elegans and $C$. briggsae (Table 1).

\section{Founding, maintenance and assessment of ecological communities}

We age-synchronized founding individuals at the first larval (L1) stage from each strain using a standard bleaching protocol [46], then estimated L1 density for each strain from three aliquots of $5 \mu \mathrm{L}$. By adjusting the concentration appropriately, we transferred $20 \mu \mathrm{L}$ drops of L1s to found each community type with $\sim 50$ or $\sim 100$ individuals of a given strain (Table 2). We varied the starting densities among community types to match the number of reproducing hermaphrodites or females for each community, assuming a 1:1 sex ratio among L1 animals for dioecious strains. We maintained populations following modified standard C. elegans procedures [47], rearing at $25{ }^{\circ} \mathrm{C}$ on E. coli (OP50) food on $9 \mathrm{~cm}$ diameter NGM-lite agar Petri dishes with agar concentration increased to $2.2 \%$ to discourage animals from burrowing [46]. To ensure communities would have an over-abundance of food for a week, we pelleted concentrated overnight $1 \mathrm{~L}$ B-broth cultures of $E$. coli by centrifugation of $50 \mathrm{~mL}$ aliquots at $5000 \mathrm{rpm}$ for $10 \mathrm{~min}$. The concentrated E. coli was re-suspended in $500 \mu \mathrm{L}$ and spread onto the surface of $9 \mathrm{~cm}$ diameter agar plates, allowed to dry, and then grown overnight at $37{ }^{\circ} \mathrm{C}$. After adding a founding community to an individual agar dish with food, Petri dishes were then sealed with Parafilm ${ }^{\circledR}$ and placed in plastic boxes with moist Kimwipes ${ }^{\circledR}$ to maintain humidity at $90-100 \%$. We initiated 10 replicates per treatment (with two exceptions, one treatment with $\mathrm{n}=9$ and one with $\mathrm{n}=12$ ).

We conducted just a single transfer of communities to fresh food, after 7 days ( 3 generations), to minimize disruption to communities. None of the replicates had depleted their food supply or gone extinct prior to transfer and all replicates retained all strains that they started with, distinguishable by fluorescent markers. We then estimated animal abundance to calculate intrinsic growth rates for single-genotype communities according to an exponential growth model (see calculations below): after washing worms off each plate with $5 \mathrm{~mL}$ M9 buffer, we estimated density (worms $/ \mu \mathrm{L}$ ) from a $2 \mu \mathrm{L}$ aliquot. Simultaneously, we transferred $20 \mu \mathrm{L}$ from each replicate to a new $9 \mathrm{~cm}$ agar plate with food as described above (aliquot of $0.4 \%$ ).

We terminated the experiment 15 days after inception ( $\sim 6$ generations; no extinctions), estimating densities and the proportional composition of distinct strains within each replicate community. As previously, we estimated density (worms $/ \mu \mathrm{L}$ ) from the average of three aliquots of $2 \mu \mathrm{L}$ from a $5 \mathrm{~mL}$ wash of each dish with M9 buffer. We then concentrated the worms (1800 rcf for $2 \mathrm{~min}$ ) and pipetted a $2 \mu \mathrm{L}$ aliquot and $2 \mu \mathrm{L}$ of $500 \mathrm{mM}$ of sodium azide (to induce paralysis) onto a microscope slide with a $10 \%$ agarose pad, covered by a glass coverslip. We captured digital images of worms with white light and with red and green fluorescent channels at $4 \times$ or $10 \times$ magnification (depending on spatial distribution of worms) with a fluorescence compound microscope and

Table 2 Initial experimental composition of each community type

\begin{tabular}{|c|c|c|c|}
\hline Phenotypes of C. elegans or C. briggsae ${ }^{a}$ & Frequency of wildtype (\%) & Frequency of C. nigoni (\%) & $\begin{array}{l}\text { Number of individuals } \\
\text { in founding community }\end{array}$ \\
\hline Wildtype hermaphrodites & 100 & 0 & 50 \\
\hline None & 0 & 100 & 100 \\
\hline Wildtype hermaphrodites & 33 & 67 & 150 \\
\hline Vulvaless hermaphrodites & 0 & 67 & 150 \\
\hline Feminized females and males & 0 & 50 & 200 \\
\hline Wildtype and vulvaless hermaphrodites & 50 & 0 & 100 \\
\hline $\begin{array}{l}\text { Wildtype hermaphrodites and feminized females and } \\
\text { males }\end{array}$ & 33 & 0 & 150 \\
\hline
\end{tabular}

\footnotetext{
${ }^{a}$ Each of the seven community types were initiated for blocks with C. elegans or with C. briggsae

b $C$. nigoni were excluded from three community types that contained only wildtype hermaphrodites or mixtures of conspecific genotypes with contrasting reproductive modes

c Initial population sizes were set to equalize the number of egg-laying individuals (females or hermaphrodites) to 50 for each species within each community type assuming 1:1 sex ratio for genotypes with males and females
} 
camera (Olympus BX51 with DP80 camera, using Olympus cellSens Standard 1.14). We then manually marked the location of worms of each fluorescent phenotype on each image using Adobe Photoshop CS6 (minimum 100 worms per replicate). We counted the number of worms of each type by processing the marked images using Image)'s particle analysis function. In 3 of the 102 replicates of two-strain communities, one of the strains was not detected, despite both types being visible in qualitative scans of each replicate sample. Consequently, we used a pseudo-count of 1 for the rarer type in subsequent calculations for those three replicates.

We noticed that not all worms showed detectable expression of the DsRed marker in those communities comprised solely of $C$. nigoni that should show $100 \%$ of red individuals: $87.1 \% \pm 1 \mathrm{SE}=2.13$ (experimental block with $C$. briggsae, $n=9$ ), $80.3 \% \pm 2.10$ (experimental block with $C$. elegans, $n=10)$. By contrast, nearly all individuals expressed GFP $(97.9 \% \pm 6.38$ in C. briggsae, $n=10$; $99.8 \% \pm 0.0013$ in C. elegans, $n=10$ ). Consequently, we numerically adjusted the relative abundance accordingly for $C$. nigoni in mixed communities to account for this imperfect detection, using a correction factor separately for the experimental blocks conducted with $C$. elegans and with C. briggsae.

\section{Growth rate estimation in ecological communities}

We estimated the "community rate of growth" of the population mixtures for each replicate by solving for $r$ in a simple continuous time model for exponential population growth, which assumes unlimited resources, using estimates of initial and final abundances. Specifically, given the starting number of worms in a treatment $\left(C_{0}\right)$ and the number after $t$ generations $\left(C_{t}\right)$ :

$$
C_{t}=C_{0} e^{r t}
$$

Solving for the intrinsic rate of growth $(r)$ yields:

$$
r=\frac{\ln \left(\frac{C_{t}}{C_{0}}\right)}{t}
$$

We used the input number of founding individuals for the value of $C_{0}$ and the number of worms estimated at the transfer point for $C_{t}$, where we assume $t=3$ generations. An ANOVA was then performed to test for differences in growth rate $(r)$ among treatments. For single-strain communities, $r$ represents the intrinsic rate of population growth for that strain; for multi-strain communities, $r$ represents a metric of biomass accumulation as a combined measure of community-wide population growth across strains or species.

We could calculate $r$ directly for single-strain treatments only for $C$. nigoni and for wildtype $C$. elegans and C. briggsae. To estimate growth rate $(r)$ for individual strains in mixed-strain communities that had geneticallytransformed reproductive modes (obligatorily dioecious, obligately selfing), we used information about the relative abundances of each strain at the inception and end of the experiment in a model of exponential growth. Specifically, we related the relative abundances given frequency $P_{0}$ of the competitor strain when the community was founded and the final frequency $P_{t}$ of the competitor strain at the end of the experiment as

$$
\frac{P_{t}}{1-P_{t}}=\frac{P_{0} e^{r t}}{\left(1-P_{0}\right) e^{r^{\prime} t}}
$$

(frequencies $1-P_{0}$ and $1-P_{t}$ for the focal strain; $t=6$ generations). We then computed the estimated growth rate for the focal strain by solving for $r^{\prime}$ to yield:

$$
r^{\prime}=\left[r t+\ln \left(\frac{P_{0}\left(1-P_{t}\right)}{\left(1-P_{0}\right) P_{t}}\right)\right] / t
$$

Using this approach, we estimated the growth rate of individual strains in each replicate for treatments of mixed-strain communities that contained obligate selfing (vulvaless) or obligate dioecious (feminized hermaphrodites) reproductive modes.

\section{Statistical analyses for ecological experiments}

Statistical analyses were performed using IBM SPSS Statistics for Windows, Version 20.0 (2011). For statistical analyses, $\mathrm{n}$ was defined as the number of replicate community populations. Independent-sample t-tests were applied to test for differences in growth rates among community types, using Bonferroni correction for multiple tests when examining the growth rates involving pure wildtype strains of each C. elegans, $C$. briggsae and C. nigoni $(\alpha=0.025$; Fig. 1$)$. One-sample $t$-tests were applied to arcsine transformed data to test for differences between the initial and final frequencies of individual strains in mixed strain communities. An ANOVA was applied to test for differences in population density among community types.

\section{Results}

To test whether harm induced by $C$. nigoni male sperm affects other species at the demographic level, we assessed populations of C. elegans or C. briggsae mixed with $C$. nigoni. By inferring species relative abundance and growth rate ( $r$ in Eq. 2 or $r^{\prime}$ in Eq. 4) under conditions 

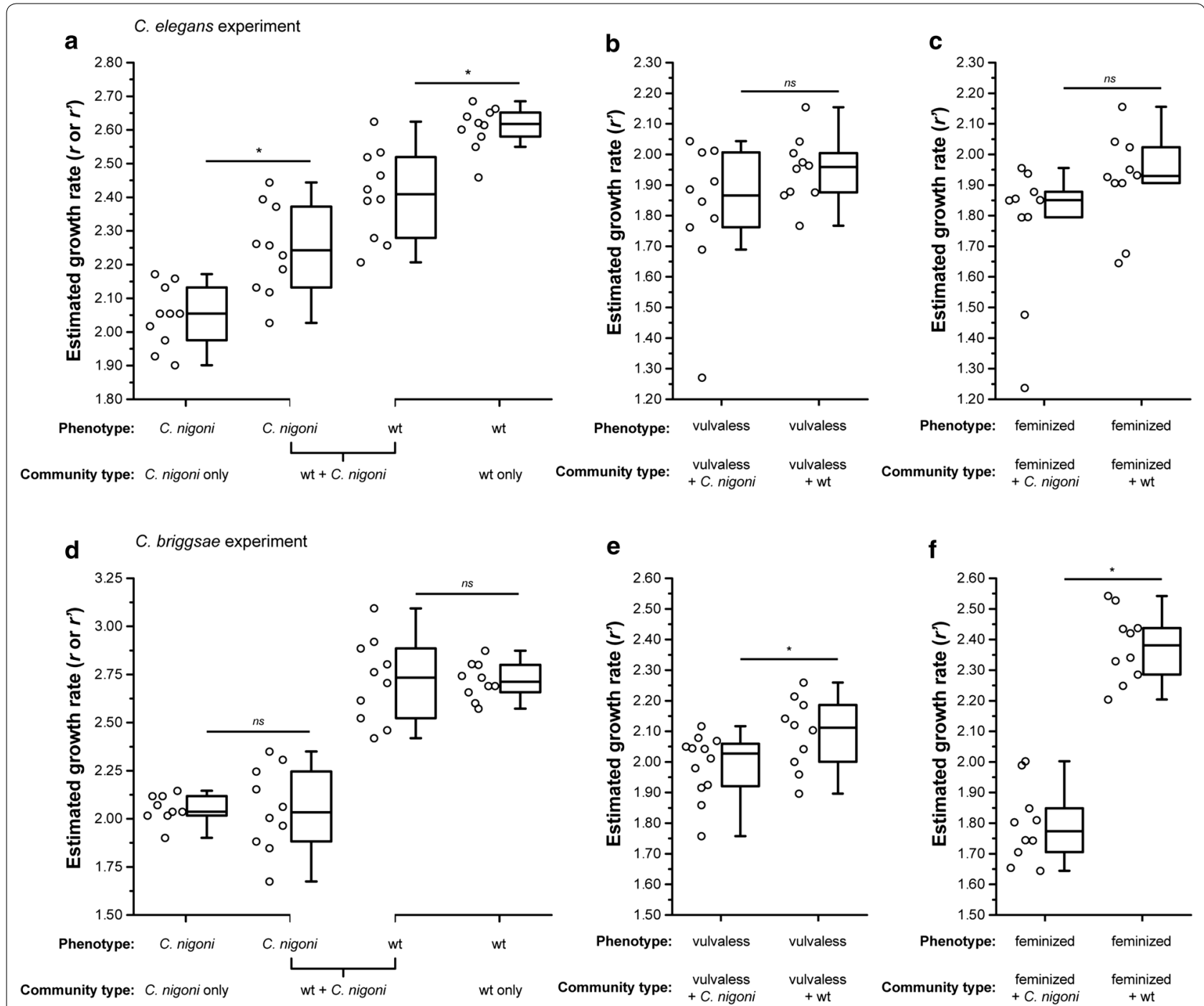

Fig. 1 Presence of C. nigoni interferes with reproduction of C. elegans and C. briggsae. a-c Growth rate estimates for the C. elegans experimental block show significant differences in wildtype $C$. elegans (wt) growth rate when grown alone or in the presence of $C$. nigoni, but no effect of $C$. nigoni on growth rate estimates for vulvaless and feminized strains of $C$. elegans. $\mathbf{d}-\mathbf{f}$ In the C. briggsae experimental block, growth rate estimates for both vulvaless and feminized strains of $C$. briggsae are reduced in the presence of $C$. nigoni, whereas wildtype $C$. briggsae growth rates were not significantly affected. Growth rate estimates for each species or reproductive phenotype in mixed communities ( $\left.r^{\prime}\right)$ used Eq. 4 that incorporates direct growth rate estimates $(r)$ from pure strains based on Eq. 2. The growth rates from the different community types were compared using a $t$ test with Bonferroni correction for multiple tests in (a wt comparison $t=4.22, \mathrm{df}=18, \mathrm{P} \leq 0.001$; C. nigoni comparison $t=-3.86, \mathrm{df}=18, \mathrm{P} \leq 0.001$ ) and ( $\mathbf{d}$ wt comparison $t=-0.03, \mathrm{df}=12.25, \mathrm{P}=0.98 ; \mathrm{C}$. nigoni comparison $t=0.03, \mathrm{df}=11.25, \mathrm{P}=0.98)(\mathrm{a}=0.025)$, where asterisks $\left.{ }^{*}\right)$ indicate differences $(a=0.05)$ and $n$ indicates non-significant differences $(\mathbf{b} t=-1.59, \mathrm{df}=18, \mathrm{P}=0.13 ; \mathbf{c} t=-1.76, \mathrm{df}=18, \mathrm{P}=0.095 ; \mathbf{e} t=-2.22, \mathrm{df}=20$, $\mathrm{P}=0.038 ; \mathbf{f} t=-10.91, \mathrm{df}=18, \mathrm{P} \leq 0.001$ ). Boxplot whiskers indicate $1.5^{*}$ (interquartile range) and open circles show estimates for each replicate population

of non-limiting resources for these communities across six generations, we tested for 'demographic RI'. Moreover, we genetically manipulated the mode of reproduction for C. elegans and C. briggsae to create three distinct treatments that differed in their likelihood of receiving male sperm (high for a feminized obligate dioecious strain, moderate for wildtype hermaphrodites, and low for vulvaless obligate selfing hermaphrodites) to explore how sensitive is the response to this gametic mechanism of interference competition to the risk of heterospecific sperm exposure.

First, we confirmed that the intrinsic population growth rate of the obligately outcrossing species $C$. nigoni is lower than the population growth rate of both 
C. elegans and C. briggsae when each species was grown in isolation $(C$. elegans: $\mathrm{t}=15.7, \mathrm{df}=18, \mathrm{P} \leq 0.001 ; C$. briggsae: $\mathrm{t}=17.0, \mathrm{df}=17, \mathrm{P} \leq 0.001$; Fig. $1 \mathrm{a}, \mathrm{d})$. This result affirms that $C$. elegans and $C$. briggsae should be superior resource competitors than $C$. nigoni if resources were limiting. We also saw that wildtype hermaphrodites of $C$. briggsae had a higher growth rate than $C$. elegans $(t=3.05, d f=18, P=0.007)$, consistent with the experimental temperature $\left(25{ }^{\circ} \mathrm{C}\right)$ being more favourable for C. briggsae [48]. Communities that consisted purely of C. nigoni showed similar growth rates in experiments involving both $C$. briggsae and $C$. elegans (ANOVA: $\mathrm{t}=0.17, \mathrm{df}=17, \mathrm{P}=0.87$; Fig. $1 \mathrm{a}, \mathrm{d})$ and reached similar densities (Additional file 1: Figure S1). That C. nigoni imposes component RI on C. elegans and C. briggsae, but not the reciprocal [28], motivated us to test whether reproductive interference might partly or completely offset the direct competitive disadvantage of $C$. nigoni.

We next sought to determine whether interspecies interactions in mixed assemblages of species would change the species-specific growth rate estimates when food was not limited, thus revealing competitive interference. When mixed with $C$. nigoni, the $C$. elegans strain with the wildtype hermaphrodite reproductive mode showed significantly slower population growth than when reared in isolation $\left(\mathrm{F}_{3,36}=47.63, \mathrm{P} \leq 0.001 ; \mathrm{t}=4.22\right.$, $\mathrm{df}=18, \mathrm{P} \leq 0.001$; Fig. $1 \mathrm{a}, \mathrm{d})$. This result is consistent with $C$. elegans suffering from demographic reproductive interference by $C$. nigoni males. Despite the reduced population growth rate of $C$. elegans wildtype hermaphrodites mixed with $C$. nigoni, they still increased in relative frequency over time, indicating that the negative interspecies interaction was not sufficient to fully offset the intrinsic growth advantage of $C$. elegans over $C$. nigoni given their relative starting frequencies (Fig. 2a; Additional file 1: Figure S2A). We also observed significantly faster population growth of $C$. nigoni in the mixedspecies community with $C$. elegans than when grown alone $(\mathrm{t}=-3.86, \mathrm{df}=18, \mathrm{P} \leq 0.001$; Fig. $1 \mathrm{a})$, suggesting the possibility of facilitation by an unknown mechanism.
Mixed species communities of $C$. nigoni with $C$. briggsae that had the wildtype hermaphrodite reproductive mode, however, exhibited no difference in population growth rate for either species compared to when they were reared in isolation $(C$. briggsae $\mathrm{t}=-0.03, \mathrm{df}=12.25$, $\mathrm{P}=0.98 ; C$. nigoni $\mathrm{t}=0.03, \mathrm{df}=11.25, \mathrm{P}=0.98$; Fig. $1 \mathrm{~d})$. This result implies that wildtype $C$. briggsae did not suffer from demographic reproductive interference by $C$. nigoni males, despite strong 'component RI' effects [28].

We hypothesized that if mating is obligatory for reproduction, then a species would be more likely to experience interspecies errors in mating and be exposed to the potential influence of harmful gametic effects from C. nigoni. Consequently, we constructed species assemblages of $C$. nigoni mixed with feminized populations of C. elegans or C. briggsae that had been genetically manipulated to be composed of females and males that reproduce via obligate outcrossing instead of by self-fertilizing hermaphrodites. Estimates of the population growth rate for these feminized $C$. briggsae populations were significantly slower when grown with $C$. nigoni than when feminized $C$. briggsae were grown with wildtype hermaphrodite $C$. briggsae $(\mathrm{t}=-10.91, \mathrm{df}=18, \mathrm{P} \leq 0.001$; Fig. 1f). This observation is consistent with demographic RI by $C$. nigoni males reducing $C$. briggsae growth rates under conditions where $C$. briggsae must mate in order to reproduce. However, feminized $C$. elegans only showed a non-significant trend of reduced population growth rates by the presence of $C$. nigoni $(\mathrm{t}=-1.76, \mathrm{df}=18, \mathrm{P}=0.095$; Fig. 1c), suggesting a weaker magnitude of effect on $C$. elegans than C. briggsae.

Finally, we hypothesized that a species incapable of being inseminated would be less susceptible to demographic RI by harmful effects of gametes from another species. Therefore, we constructed species assemblages with $C$. nigoni mixed with populations of C. elegans and C. briggsae that were genetically manipulated so that hermaphrodites lacked a vulva and so could not be inseminated; vulvaless animals reproduce obligately by selfing. Indeed, we observed no significant difference in our

\footnotetext{
(See figure on next page.)

Fig. 2 Relative frequencies of species and strain phenotypes changed in communities over time. Frequencies of species and strain phenotypes in mixed communities after six generations for experimental blocks with C. elegans ( $\mathbf{a}, \mathbf{b})$ and C. briggsae (c, d). Wildtype (wt) strains of C. elegans increased in frequency regardless of which other strain phenotype or species they were paired with (a community mix with vulvaless $t(9)=42.07$, $\mathrm{P} \leq 0.001$; C. nigoni $(9)=10.19, \mathrm{P} \leq 0.001$; feminized $t(9)=30.57, \mathrm{P} \leq 0.001$ ), as was also true for $C$. briggsae (c community mix with vulvaless $t(9)=37.71, P \leq 0.001$; C. nigoni $t(9)=18.02, P \leq 0.001$; feminized $t(9)=10.82, P \leq 0.001)$. By contrast, the feminized phenotype of both $C$. elegans and C. briggsae declined in frequency when grown with C. nigoni (b C. elegans $t(9)=-5.82, P \leq 0.001 ; \mathbf{d}$ C. briggsae t(9) $=-7.12, P \leq 0.001)$. We observed lower frequencies of vulvaless C. elegans hermaphrodites when mixed with C. nigoni, as well $(\mathbf{b} t(9)=-4.23, P=0.002)$, although the relative frequency of vulvaless C. briggsae was not significantly reduced over time when reared with C. nigoni $(\mathbf{d} t(11)=-2.11, \mathrm{P}=0.059)$. Asterisks $\left(^{*}\right)$ indicate significant differences $(a=0.05)$ between starting phenotype frequencies (dashed lines) and ending phenotype frequencies (ns indicates $\mathrm{P}>0.05$ ) from one-sample t-tests after arcsin transform. The values shown for the "wt $+C$. nigoni" community type in $\mathbf{a}$ and $\mathbf{b}$ correspond to the same underlying data and test of significance; values for "wt + C. nigoni" in c and $\mathbf{d}$ correspond to the same data and test of significance
} 

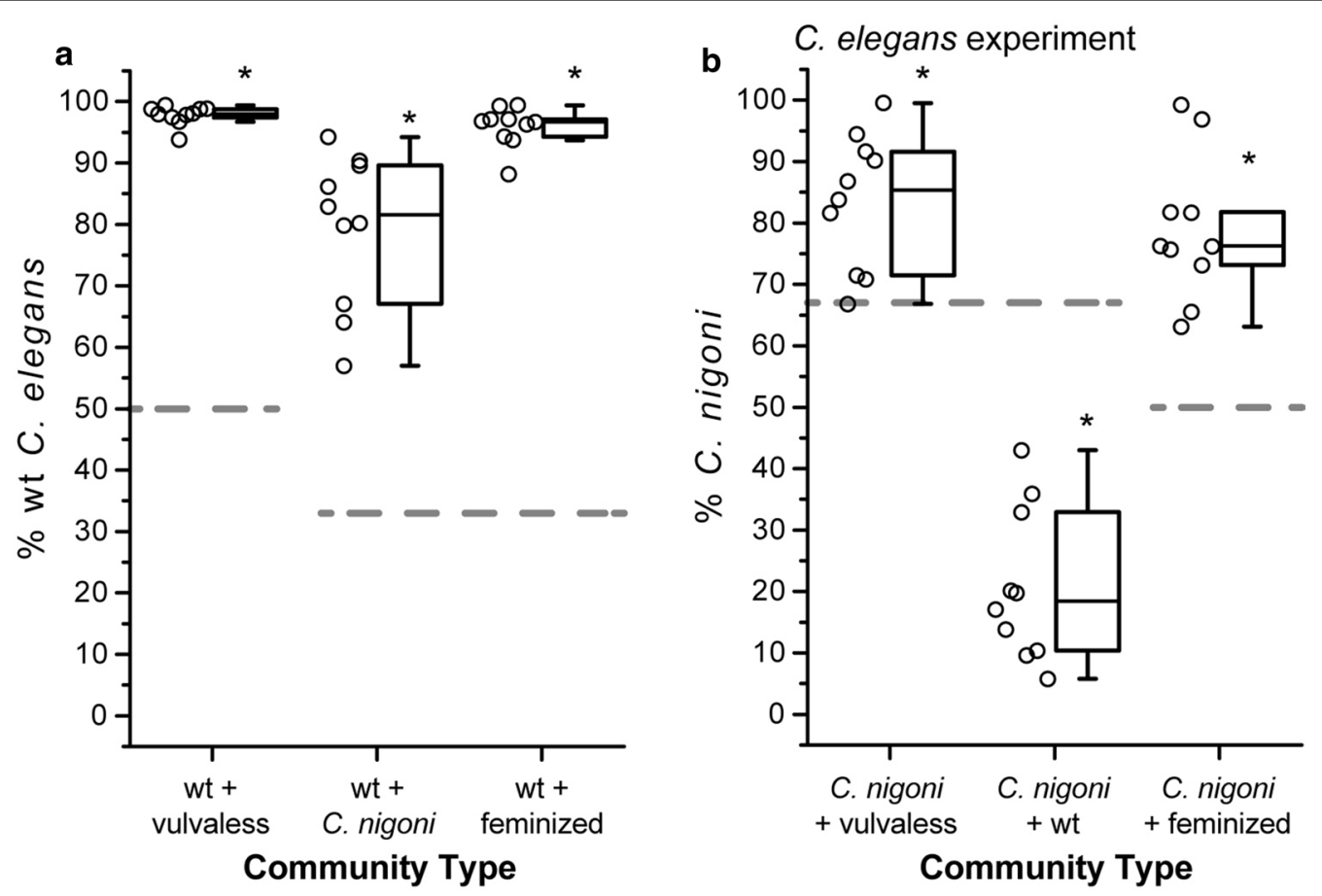

\section{Community Type}

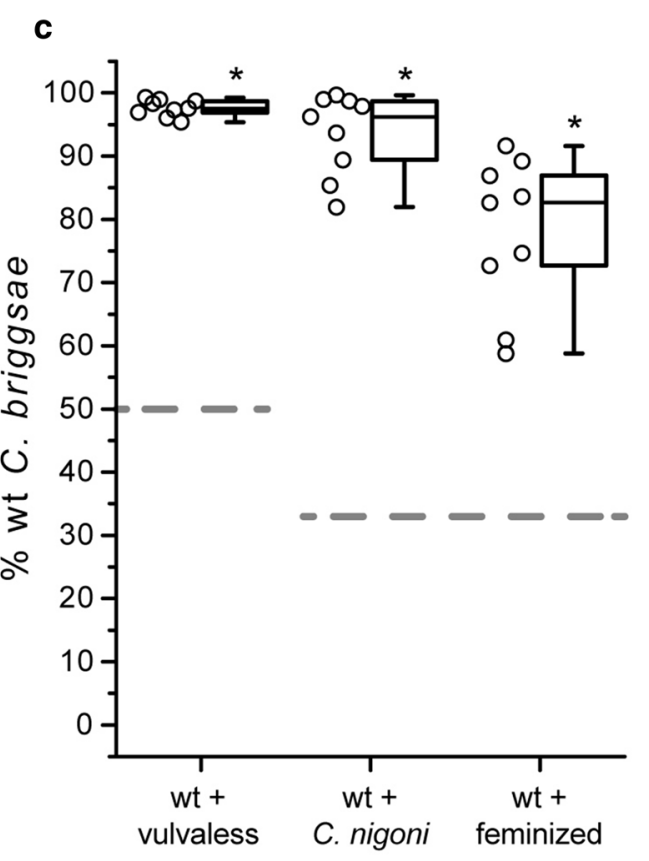

Community Type

d C. briggsae experiment

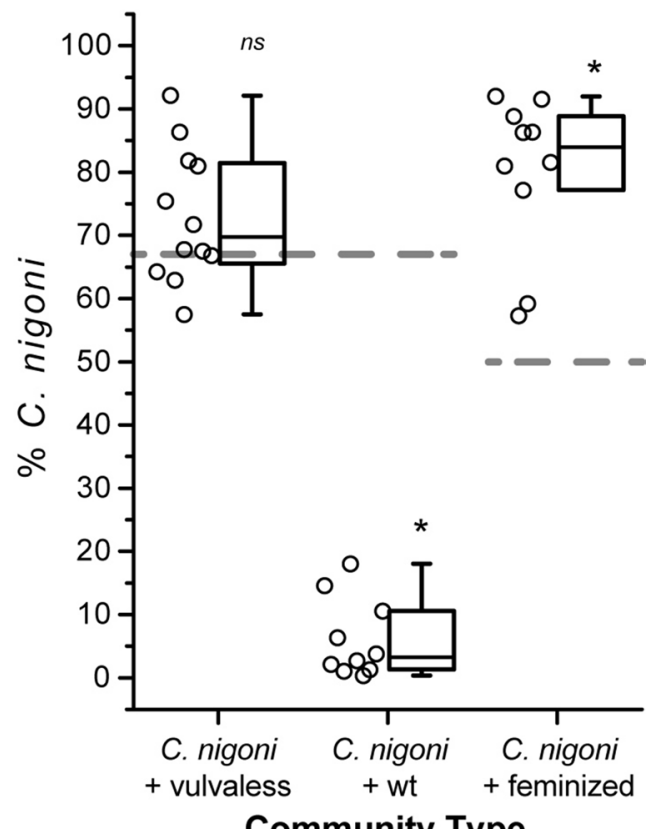

Community Type estimates of population growth rate for vulvaless C. elegans when mixed with $C$. nigoni versus when grown with wildtype hermaphrodite $C$. elegans $(\mathrm{t}=-1.59, \mathrm{df}=18$, $\mathrm{P}=0.13$; Fig. 1b). Estimates of population growth rate for vulvaless $C$. briggsae, by contrast, were significantly lower in the presence of $C$. nigoni than when reared with wildtype $C$. briggsae $(\mathrm{t}=-2.22, \mathrm{df}=20, \mathrm{P}=0.038$; Fig. 1e). This latter observation suggests that some form of competitive interference mediated by a mechanism other than $C$. nigoni sperm influenced the fitness of 
vulvaless $C$. briggsae individuals. The proportion of vulvaless (and feminized) C. elegans strains declined from initial frequencies when in presence of either conspecifics or dioecious C. nigoni (Fig. 2a, b; Additional file 1: Figure $\mathrm{S} 2 \mathrm{~A}$ ), with the disadvantage of dioecious $C$. elegans relative to hermaphrodite C. elegans recapitulating previous findings $[49,50]$. Similarly, the proportion of feminized C. briggsae also declined from initial frequencies when in the presence of conspecifics or dioecious C. nigoni (Fig. 2b), whereas vulvaless C. briggsae hermaphrodites only declined significantly when mixed with wildtype conspecifics (Fig. 2b).

\section{Discussion}

\section{Gametic vs. non-gametic interference competition}

Our multi-species experimental communities demonstrate that the population growth rate of a given species can suffer from the presence of another species, even under conditions of non-limiting resource availability. These findings implicate some form of interference competition mediating interspecies interactions, with Caenorhabditis nematode biology pointing to reproductive interference as the predominant source [10, 28, 51]. We designed the communities of Caenorhabditis to include species combinations with inherent asymmetry in their resource competitive ability versus ability to induce harm through reproductive interactions. In particular, "component reproductive interference (RI)" by C. nigoni males to individual hermaphrodites of $C$. elegans and $C$. briggsae induces sterility and premature death, mediated by ectopic sperm cell migration throughout the gonad and body cavity upon interspecies matings, capable of reducing lifetime fitness by $\sim 85 \%$ [28]. Consequently, we conclude that this mechanism of component RI ramifies to the population level, with "demographic reproductive interference" best explaining our observations of reduced growth rates observed in C. elegans and C. briggsae when they share experimental environments with C. nigoni.

In one experimental treatment, however, we detected evidence of interference competition that cannot be explained by gamete-mediated RI: populations of $C$. briggsae incapable of mating suffered reduced growth rates in the presence of $C$. nigoni. This result contrasts with our initial prediction that reduced risk of invasive heterospecific sperm would eliminate the potential for reproductive interference. We hypothesize that excessive but futile male mating attempts by $C$. nigoni on the vulvaless $C$. briggsae hermaphrodites reduced their fitness by direct physical damage or by interfering with foraging and feeding. Male nematodes continuously trace the body of a potential mating partner in an attempt to locate the vulva for spicule insertion and insemination ('circling behavior') [32, 52], leading to cuticular damage and, potentially, attempted erroneous copulation with the excretory pore [27, 31, 53, 54]. Moreover, as eggs hatch inside a vulvaless animal, her locomotion becomes compromised which could hamper normal hermaphrodite ability to evade male mating attempts $[27,45,55]$, thus exacerbating the potential negative direct effects of persistent male courtship. Continual hermaphrodite escape behavior in the face of persistent male courtship also would limit foraging, thus also compromising resource uptake. Secretions by Caenorhabditis males also have been shown to alter the physiology and reduce the longevity of conspecific hermaphrodites, even in the absence of any physical interactions $[30,56]$. Consequently, such non-gametic forms of pre-copulatory reproductive interference could explain the reduced growth rate of vulvaless $C$. briggsae in the presence of $C$. nigoni and might also contribute a source of interference competition in other circumstances as well.

We observed distinct responses to the presence of $C$. nigoni for $C$. elegans and C. briggsae when subjected to analogous mating system treatments. Given that selfing hermaphroditism evolved independently in each of these species, the distinct effects may reflect independently evolved tactics in response to male mating attempts and sperm activity [57-60]. C. briggsae is more closelyrelated to C. nigoni than is C. elegans [60]. Moreover, the geographic range of $C$. briggsae overlaps that of tropical C. nigoni, unlike for the temperate range of C. elegans; encounters with heterospecific Caenorhabditis might be more common in nature for tropical species in general because Caenorhabditis are more speciose in the tropics [33, 60, 61]. Consequently, we might expect stronger reproductive character displacement to have evolved in C. briggsae in the form of mate discrimination or other pre-mating isolation barriers [7]. If present, then stronger pre-mating barriers to interspecies mating could explain the lack of evidence for demographic RI for wildtype $C$. briggsae and its presence for the treatment with obligatory outbreeding C. briggsae (and the converse pattern for $C$. elegans). The pre-mating barriers might be insufficient to offset the exposure to greater risk of mating with heterospecifics for the obligately outbreeding C. briggsae treatment, making the populations experience a greater influence of the strong component RI once interspecies mating took place. While matings can occur readily between many Caenorhabditis species, few quantitative data have tested the degree to which pre-mating barriers might modulate inter-species mating risk $[28,62,63]$.

Future work that formally tests for responses of species pairs with allopatric versus sympatric distributions would prove valuable in testing for the generality of reproductive interference to mediate reproductive character displacement and pre-mating reproductive isolation. As 
yet, however, few studies have demonstrated much evidence of mate choice among Caenorhabditis species $[28,62,63]$. Similarly, the phylogeographically-partitioned genetic variation in C. briggsae could prove fruitful in describing the genetic basis to any within-species genetic variation in pre-mating barriers or in susceptibility to sperm invasion $[64,65]$. It also will be important to determine whether sperm as a mechanism of interspecies harm represents only an incidental weapon, or whether gametic reproductive interference has been co-opted more actively as an adaptive trait mediating competitive encounters between species (cf. allelopathy between plant species [66]).

\section{Frequency dependence in RI}

Our experiments initialized communities with similar relative abundances of the species. However, the potential for demographic RI to control the dynamics of community composition is stronger for more skewed relative abundances, due to its frequency dependent effects [13, $15,67]$. This feature essentially describes a 'priority effect', a common feature of ecological communities whereby the attributes of established individuals determine the ability for later arrivals to invade or coexist [e.g. 15, 68-70]. As a result, it should be easier to detect demographic RI when the starting frequencies of species are highly skewed because fitness loss from RI will be amplified when the rarer species experiences proportionally more harmful heterospecific encounters [70-72]. Consequently, our experimental design is conservative with respect to being able to detect demographic RI, which likely explains why we observed demographic RI for only some experimental treatments. Future work that disentangles the potential influences of frequency dependent effects, pre-mating barriers, and species differences will be valuable in fully deciphering the importance of demographic RI in mediating species coexistence.

Importantly, previous findings of component RI in Caenorhabditis generally used highly male skewed sex ratios (1 hermaphrodite: 6 heterospecific males) and only gave $C$. nigoni males the option of mating with heterospecific females, which could inflate the incidence of interspecies mating relative to when a choice of conspecific versus heterospecific mates are available [28]. Even those conditions favorable to interspecies mating yielded $<50 \%$ interspecies mating success after $18-24 \mathrm{~h}$ (unpublished observations), reflecting the facts that $C$. nigoni females are more likely than $C$. briggsae and $C$. elegans hermaphrodites to mate with $C$. nigoni males [28] and that hermaphrodites are behaviourally uncooperative, even with conspecific males [27]. Despite these factors that should limit our ability to detect demographic reproductive interference, we nevertheless observed repeated cases of reduced population growth rates of $C$. elegans and C. briggsae when they co-occurred with $C$. nigoni. Future studies with Caenorhabditis that build on our proof-of-principle demonstration of demographic RI should further characterize it by testing additional species communities and by varying the relative abundance in the composition of founding communities [70].

Although our experimental design largely precludes the possibility of evolution, we anticipate that sperm-mediated reproductive interference could drive trait evolution within species. Future studies with Caenorhabditis can address directly this issue [73]. Specifically, we expect that the strength of reproductive interference, and correspondingly the strength of selection, would be greater when one species is rarer because of more intense exposure to harmful interactions, provided that the cost is not so great as to drive it locally extinct. As for other mechanisms of reproductive character displacement [24], we should expect more frequent encounters between species that exact sperm-mediated harm during sexual interactions to lead to the faster evolution of stronger pre-mating reproductive isolation [74]. It remains unclear how sensitive to encounter frequency would be the coevolutionary dynamics between species in causing and evading harm from reproductive interference. While sympatryallopatry comparisons of reproductive traits provide a classic way to evaluate the consequences of interspecies interactions, quantitative variation in the likelihood of interspecies encounters present a greater challenge for prediction [74]. Theory shows that even small amounts of harmful reproductive interactions between species can confer large effects on trait evolution and coexistence relative to direct resource competition [74]. Caenorhabditis provides an experimentally tractable system for future tests of additional features of coevolutionary and coexistence theory. These basic principles generalize to systems beyond nematodes and their particular mechanism of reproductive interference [7-9], permitting experimental interrogation of theory about ecoevolutionary processes.

\section{Conclusion}

Our laboratory experiments with Caenorhabditis nematodes demonstrate how interspecies reproductive interference between individuals can ramify to influence population demography, with the potential to alter species community structure and trait evolution. The mechanisms of reproductive interference involve negative effects of ectopic sperm migration throughout the female or hermaphrodite body [28], with a potential additional contribution of physical harm and food deprivation from continuous escape behavior induced by heterospecific mating attempts [27, 31]. It remains unknown, however, how frequently species of Caenorhabditis interact with 
one another in nature. The intensifying study of the natural context of Caenorhabditis nematodes [33, 37, 48, 61, $75,76]$, along with naturalistic experimental microcosms $[73,77]$, make them promising for relating species coexistence theory, mechanisms of reproductive interference, antagonistic coevolution and reproductive character displacement, and community dynamics in nature. With rapidly changing and human-impacted environments around the world, organisms that have historically never interacted with one another are being brought together at an unprecedented pace [78], including invasive species with small initial populations relative to native population sizes [79]. Therefore, full consideration of reproductive interference as a mode of interspecies interactions is crucial for elucidating a general understanding of biodiversity and ecological functioning, with Caenorhabditis offering a powerful system for manipulative study.

\section{Additional files}

Additional file 1: Figure S1. Density of communities at the end of the experiment. Figure S2. Change in the relative frequency of phenotypes or species in different community types.

Additional file 2. Raw data for Caenorhabditis reproductive interference experiments.

\section{Abbreviations}

Rl: reproductive interference; GFP: green fluorescent protein

\section{Authors' contributions}

JJT and ADC designed research; JJT performed research; ADC contributed reagents/analytic tools; JJT analyzed data; JJT and ADC wrote and edited the manuscript. Both authors read and approved the final manuscript.

\begin{abstract}
Acknowledgements
We thank Jeremy Gray for his molecular and experimental evolution expertise during the study. We thank Joel Levine, Helen Rodd, and Jonathan Schneider for their input on the experimental design. We thank Bhagwati P. Gupta, MarieAnne Félix, Ronald E. Ellis and the CGC for providing strains (CGC funded by $\mathrm{NIH}$ Office of Research Infrastructure Programs: P40 OD010440).
\end{abstract}

\section{Competing interests}

The authors declare that they have no competing interests.

\section{Availability of data and materials}

Data used for analysis is provided in the supplement, strains are publicly available from the Caenorhabditis Genetics Center. The datasets supporting the conclusions of this article are included within the article and its Additional files 1,2 .

\section{Consent to publish}

Not applicable.

\section{Ethics approval and consent to participate} Not applicable.

\section{Funding}

ADC is supported by Discovery Grants from the Natural Sciences and Engineering Research Council of Canada.

\section{Publisher's Note}

Springer Nature remains neutral with regard to jurisdictional claims in published maps and institutional affiliations.

Received: 28 April 2018 Accepted: 26 October 2018

Published online: 06 November 2018

\section{References}

1. Goldberg DE, Barton AM. Patterns and consequences of interspecific competition in natural communities: a review of field experiments with plants. Am Nat. 1992;139(4):771-801.

2. Stachowicz JJ. Mutualism, facilitation, and the structure of ecological communities: positive interactions play a critical, but underappreciated, role in ecological communities by reducing physical or biotic stresses in existing habitats and by creating new habitats on which many species depend. Bioscience. 2001;51(3):235-46.

3. Sih A, Crowley P, McPeek M, Petranka J, Strohmeier K. Predation, competition, and prey communities: a review of field experiments. Annu Rev Ecol Syst. 1985;16(1):269-311.

4. Chase JM, Abrams PA, Grover JP, Diehl S, Chesson P, Holt RD, Richards SA, Nisbet RM, Case TJ. The interaction between predation and competition: a review and synthesis. Ecol Lett. 2002;5(2):302-15.

5. Chamberlain SA, Bronstein JL, Rudgers JA. How context dependent are species interactions? Ecol Lett. 2014;17(7):881-90.

6. Mendelson TC, Shaw KL. The (mis) concept of species recognition. Trends Ecol Evol. 2012;27(8):421-7.

7. Gröning J, Hochkirch A. Reproductive interference between animal species. O Rev Biol. 2008:83(3):257-82.

8. Burdfield-Steel ER, Shuker DM. Reproductive interference. Curr Biol. 2011;21(12):R450-1.

9. Grether GF, Losin N, Anderson CN, Okamoto K. The role of interspecific interference competition in character displacement and the evolution of competitor recognition. Biol Rev. 2009;84(4):617-35.

10. Kyogoku D. Reproductive interference: ecological and evolutionary consequences of interspecific promiscuity. Popul Ecol. 2015:57(2):253-60.

11. Cothran RD. The importance of reproductive interference in ecology and evolution: from organisms to communities. Popul Ecol. 2015;57(2):339-41.

12. Chapman T, Arnqvist G, Bangham J, Rowe L. Sexual conflict. Trends Ecol Evol. 2003;18(1):41-7

13. Kuno E. Competitive exclusion through reproductive interference. Res Popul Ecol. 1992;34(2):275-84

14. Ribeiro J, Spielman A. The satyr effect: a model predicting parapatry and species extinction. Am Nat. 1986;128:513-28.

15. Yoshimura J, Clark CW. Population dynamics of sexual and resource competition. Theor Popul Biol. 1994:45(2):121-31.

16. Kishi S, Nakazawa T. Analysis of species coexistence co-mediated by resource competition and reproductive interference. Popul Ecol. 2013;55(2):305-13.

17. Stachowicz JJ, Terwin JR, Whitlatch RB, Osman RW. Linking climate change and biological invasions: ocean warming facilitates nonindigenous species invasions. Proc Natl Acad Sci. 2002:99(24):15497-500.

18. Callaway RM, Ridenour WM. Novel weapons: invasive success and the evolution of increased competitive ability. Front Ecol Environ. 2004;2(8):436-43.

19. Longcore T, Rich C. Ecological light pollution. Front Ecol Environ. 2004:2(4):191-8.

20. Ortega CP. Effects of noise pollution on birds: a brief review of our knowledge. In: Ornithological monographs vol No. 74. American Ornithologists' Union; 2012: 6-22.

21. Fitzpatrick BM, Shaffer HB. Hybrid vigor between native and introduced salamanders raises new challenges for conservation. Proc Natl Acad Sci. 2007:104(40):15793-8.

22. Pfennig KS. Facultative mate choice drives adaptive hybridization. Science. 2007:318(5852):965-7.

23. Palumbi S. Speciation and the evolution of gamete recognition genes: pattern and process. Heredity. 2009;102(1):66. 
24. Pfennig Karin S, Pfennig DW. Character displacement: ecological and reproductive responses to a common evolutionary problem. Q Rev Biol. 2009;84(3):253-76.

25. Baird SE, Sutherlin ME, Emmons SW. Reproductive isolation in Rhabditidae (Nematoda: Secernentea): mechanisms that isolate six species of three genera. Evolution. 1992;46(3):585-94.

26. Hill KL, L'Hernault SW. Analyses of reproductive interactions that occur after heterospecific matings within the genus Caenorhabditis. Dev Biol. 2001;232(1):105-14.

27. Garcia LR, LeBoeuf $B, K$ Koo P. Diversity in mating behavior of hermaphroditic and male-female Caenorhabditis nematodes. Genetics. 2007;175(4):1761-71.

28. Ting JJ, Woodruff GC, Leung G, Shin N-R, Cutter AD, Haag ES. Intense sperm-mediated sexual conflict promotes reproductive isolation in Caenorhabditis nematodes. PLoS Biol. 2014;12(7):e1001915.

29. Diaz SA, Haydon DT, Lindström J. Sperm-limited fecundity and polyandryinduced mortality in female nematodes Caenorhabditis remanei. Biol J Lin Soc. 2010;99(2):362-9.

30. Maures TJ, Booth LN, Benayoun BA, Izrayelit Y, Schroeder FC, Brunet A. Males shorten the life span of $C$. elegans hermaphrodites via secreted compounds. Science. 2014;343:541-4.

31. Woodruff GC, Knauss CM, Maugel TK, Haag ES. Mating damages the cuticle of C. elegans hermaphrodites. PLoS ONE. 2014;9(8):e104456.

32. Liu KS, Sternberg PW. Sensory regulation of male mating behavior in Caenorhabditis elegans. Neuron. 1995;14(1):79-89.

33. Cutter AD. Caenorhabditis evolution in the wild. BioEssays. 2015:37(9):983-95.

34. Hodgkin J. Exploring the envelope: systematic alteration in the sexdetermination system of the nematode Caenorhabditis elegans. Genetics. 2002;162:767-80.

35. Anderson JL, Morran LT, Phillips PC. Outcrossing and the maintenance of males within C. elegans populations. J Hered. 2010;101:S62-74.

36. Stinson KA, Campbell SA, Powell JR, Wolfe BE, Callaway RM, Thelen GC, Hallett SG, Prati D, Klironomos JN. Invasive plant suppresses the growth of native tree seedlings by disrupting belowground mutualisms. PLoS Biol. 2006;4(5):e140.

37. Frézal L, Félix M-A. The natural history of model organisms: C. elegans outside the Petri dish. Elife. 2015;4:e05849.

38. Woodruff GC, Eke O, Baird SE, Félix M-A, Haag ES. Insights into species divergence and the evolution of hermaphroditism from fertile interspecies hybrids of Caenorhabditis nematodes. Genetics. 2010;186(3):997-1012

39. Kozlowska JL, Ahmad AR, Jahesh E, Cutter AD. Genetic variation for post-zygotic reproductive isolation between Caenorhabditis briggsae and Caenorhabditis sp. 9. Evolution. 2012;66:1180-95.

40. Baird SE, Seibert SR. Reproductive isolation in the Elegans-group of Caenorhabditis. Nat Sci. 2013;5:18-25.

41. Felix MA, Braendle C, Cutter AD. A streamlined system for species diagnosis in Caenorhabditis (Nematoda: Rhabditidae) with name designations for 15 distinct biological species. PLoS ONE. 2014;9:e94723.

42. L'Hernault SW. Spermatogenesis. In: Riddle DL, Blumenthal T, Meyer BJ, Priess JR, editors. C. elegans II. New York: Cold Spring Harbor Laboratory Press; 1997. p. 271-94.

43. LaMunyon CW, Ward S. Evolution of sperm size in nematodes: sperm competition favours larger sperm. Proc R Soc B Biol Sci. 1999;266(1416):263-7.

44. Guo Y, Lang S, Ellis RE. Independent recruitment of F box genes to regulate hermaphrodite development during nematode evolution. Curr Biol. 2009;19(21):1853-60.

45. Chen J, Caswell-Chen EP. Why Caenorhabditis elegans adults sacrifice their bodies to progeny. Nematology. 2003;5(4):641-5

46. Stiernagle T. Maintenance of C. elegans. In: The C. elegans Research Community, editor. WormBook. 2006. https://doi.org/10.1895/wormb ook.1.101.1.

47. Wood WB. The nematode Caenorhabditis elegans. Cold Spring Harbor: CSHL Press; 1988

48. Félix M-A, Duveau F. Population dynamics and habitat sharing of natural populations of Caenorhabditis elegans and C. briggsae. BMC Biol. 2012;10(1):59.

49. Stewart AD, Phillips PC. Selection and maintenance of androdioecy in Caenorhabditis elegans. Genetics. 2002;160(3):975-82.
50. Cutter AD. Mutation and the experimental evolution of outcrossing in Caenorhabditis elegans. J Evol Biol. 2005;18(1):27-34.

51. Stephens PA, Sutherland WJ, Freckleton RP. What is the Allee effect? Oikos. 1999;87:185-90.

52. Yu H, Prétôt RF, Bürglin TR, Sternberg PW. Distinct roles of transcription factors EGL-46 and DAF-19 in specifying the functionality of a polycystinexpressing sensory neuron necessary for C. elegans male vulva location behavior. Development. 2003;130(21):5217-27.

53. Noble LM, Chang AS, MCNelis D, Kramer M, Yen M, Nicodemus JP, Riccardi DD, Ammerman P, Phillips M, Islam T, et al. Natural variation in plep-1 causes male-male copulatory behavior in C. elegans. Curr Biol. 2015;25(20):2730-7.

54. Gems D, Riddle DL. Genetic, behavioral and environmental determinants of male longevity in Caenorhabditis elegans. Genetics. 2000;154(4):1597-610.

55. Han M, Aroian RV, Sternberg PW. The let-60 locus controls the switch between vulval and nonvulval cell fates in Caenorhabditis elegans. Genetics. 1990;126(4):899-913.

56. Aprison Erin Z, Ruvinsky I. Sexually antagonistic male signals manipulate germline and soma of C elegans hermaphrodites. Curr Biol. 2016;26(20):2827-33.

57. Hill RC, de Carvalho CE, Salogiannis J, Schlager B, Pilgrim D, Haag ES Genetic flexibility in the convergent evolution of hermaphroditism in Caenorhabditis nematodes. Dev Cell. 2006;10(4):531-8.

58. Kiontke K, Gavin NP, Raynes Y, Roehrig C, Piano F, Fitch DH. Caenorhabditis phylogeny predicts convergence of hermaphroditism and extensive intron loss. Proc Natl Acad Sci. 2004;101(24):9003-8.

59. Nayak S, Goree J, SchedI T. fog-2 and the evolution of self-fertile hermaphroditism in Caenorhabditis. PLoS Biol. 2004;3(1):e6.

60. Kiontke K, Felix M-A, Ailion M, Rockman M, Braendle C, Penigault J-B, Fitch D. A phylogeny and molecular barcodes for Caenorhabditis, with numerous new species from rotting fruits. BMC Evol Biol. 2011;11(1):339.

61. Ferrari C, Salle R, Callemeyn-Torre N, Jovelin R, Cutter AD, Braendle C. Ephemeral-habitat colonization and Neotropical species richness of Caenorhabditis nematodes. BMC Ecol. 2017;14:43.

62. Borne F, Kasimatis KR, Phillips PC. Quantifying male and female pheromone-based mate choice in Caenorhabditis nematodes using a novel microfluidic technique. PLoS ONE. 2017;12(12):e0189679.

63. Chasnov JR, So WK, Chan CM, Chow KL. The species, sex, and stage specificity of a Caenorhabditis sex pheromone. Proc Natl Acad Sci USA. 2007;104(16):6730-5.

64. Thomas CG, Wang W, Jovelin R, Ghosh R, Lomasko T, Trinh Q, Kruglyak L, Stein LD, Cutter AD. Full-genome evolutionary histories of selfing, splitting and selection in Caenorhabditis. Genome Res. 2015;25:667-78.

65. Ross JA, Koboldt DC, Staisch JE, Chamberlin HM, Gupta BP, Miller RD, Baird SE, Haag ES. Caenorhabditis briggsae recombinant inbred line genotypes reveal inter-strain incompatibility and the evolution of recombination. PLoS Genet. 2011;7(7):e1002174.

66. Nilsson M-C. Separation of allelopathy and resource competition by the boreal dwarf shrub Empetrum hermaphroditum Hagerup. Oecologia. 1994:98(1):1-7.

67. Takakura K-I, Nishida T, Matsumoto T, Nishida S. Alien dandelion reduces the seed-set of a native congener through frequency-dependent and one-sided effects. Biol Invasions. 2009;11(4):973-81.

68. Shulman MJ, Ogden JC, Ebersole JP, MCFarland WN, Miller SL, Wolf NG. Priority effects in the recruitment of juvenile coral reef fishes. Ecology. 1983;64(6):1508-13.

69. Shorrocks B, Bingley M. Priority effects and species coexistence: experiments with fungal-breeding Drosophila. J Anim Ecol. 1994;63:799-806.

70. Kishi S, Nishida T, Tsubaki Y. Reproductive interference determines persistence and exclusion in species interactions. J Anim Ecol. 2009;78(5):1043-9.

71. Liu S-S, De Barro P, Xu J, Luan J-B, Zang L-S, Ruan Y-M, Wan F-H. Asymmetric mating interactions drive widespread invasion and displacement in a whitefly. Science. 2007;318(5857):1769-72.

72. Giga D, Canhao J. Interspecies interference of oviposition behaviour in the cowpea weevils Callosobruchus rhodesianus (Pic) and Callosobruchus maculatus (F.). Int J Trop Insect Sci. 1997;17(02):251-5.

73. Gray JC, Cutter AD. Mainstreaming Caenorhabditis elegans in experimental evolution. Proc R Soc B Biol Sci. 2014;281(1778):20133055. 
74. Goldberg EE, Lande R. Ecological and reproductive character displacement on an environmental gradient. Evolution. 2006;60(7):1344-57.

75. Félix M-A, Braendle C. The natural history of Caenorhabditis elegans. Curr Biol. 2010;20(22):R965-9.

76. Petersen C, Dirksen P, Prahl S, Strathmann EA, Schulenburg H. The prevalence of Caenorhabditis elegans across 1.5 years in selected North German locations: the importance of substrate type, abiotic parameters, and Caenorhabditis competitors. BMC Ecol. 2014;14(1):4.

77. Schulenburg H, Félix M-A. The natural biotic environment of Caenorhabditis elegans. Genetics. 2017;206(1):55.

78. Burrows MT, Schoeman DS, Richardson AJ, Molinos JG, Hoffmann A Buckley LB, Moore PJ, Brown CJ, Bruno JF, Duarte CM. Geographical limits to species-range shifts are suggested by climate velocity. Nature. 2014;507(7493):492
79. Sakai AK, Allendorf FW, Holt JS, Lodge DM, Molofsky J, With KA, Baughman S, Cabin RJ, Cohen JE, Ellstrand NC. The population biology of invasive species. Annu Rev Ecol Syst. 2001;32(1):305-32.

80. Sharanya D, Thillainathan B, Marri S, Bojanala N, Taylor J, Flibotte S, Moerman DG, Waterston RH, Gupta BP. Genetic control of vulval development in Caenorhabditis briggsae. G3 Genes Genomes Genet. 2012;2(12):1625-41

81. Edgley ML, Kelly Liu J, Riddle DL, Fire A. Chromosomes carrying GFP markers. Worm Breeder's Gazette. 1999;15(5):20.

82. Barker DM. Copulatory plugs and paternity assurance in the nematode Caenorhabditis elegans. Anim Behav. 1994;48(1):147-56.
Ready to submit your research? Choose BMC and benefit from:

- fast, convenient online submission

- thorough peer review by experienced researchers in your field

- rapid publication on acceptance

- support for research data, including large and complex data types

- gold Open Access which fosters wider collaboration and increased citations

- maximum visibility for your research: over 100M website views per year

At BMC, research is always in progress.

Learn more biomedcentral.com/submissions 